\title{
Bola fúngica dos seios paranasais: Relato de dois casos e revisão de literatura
}

\section{Fungus ball of the paranasal sinuses: Report of two cases and literature review}

\author{
Guilherme Rasia Bosi', Gustavo Lisbôa de Braga', Tobias Skrebsky de Almeida', Adriana de Carli². \\ 1) Acadêmico de Medicina da Universidade de Caxias do Sul. \\ 2) Mestre em Biotecnologia pela Universidade de Caxias do Sul. Médica otorrinolaringologista. Professora titular da Unidade de Ensino Médico Otorrino-Oftalmológica \\ da Universidade de Caxias do Sul. \\ Instituição: Universidade de Caxias do Sul. \\ Caxias do Sul / RS - Brasil. \\ Endereço para correspondência: Adriana de Carli - Rua Coronel Flores, 510 - Sala 45 - Bairro: São Pelegrino - Caxias do Sul / RS - Brasil - CEP: 95034-060. \\ Artigo recebido em 19 de Abril de 2010. Artigo aprovado em 18 de Julho de 2010.
}

\section{RESUMO}

Introdução: Bola fúngica dos seios paranasais é uma infecção não invasiva que se caracteriza por sua cronicidade, sendo a maioria relacionada com tratamento endodôntico prévio. Acomete principalmente o seio maxilar, embora todos os seios possam ser envolvidos. O principal agente etiológico é o Aspergillus spp. A tomografia computadorizada, devido às apresentações radiológicas características, sugere o diagnóstico que é realizado definitivamente através de análises histopatológicas. O tratamento padrão-ouro é a cirurgia sinusal endoscópica com antrostomia meatal média.

Objetivo: Relatar dois casos de bola fúngica dos seios paranasais e ressaltar aspectos importantes desta patologia. Relato dos Casos: Caso 1) Paciente do sexo feminino, 78 anos, apresentou-se com queixas de dor facial há 6 meses e história prévia de tratamento endodôntico. Ao exame físico constatou-se a presença de secreção purulenta em meato médio esquerdo. O Raio X apresentou velamento completo do seio maxilar esquerdo, enquanto a tomografia computadorizada mostrou lesão calcificada neste local. Realizou-se sinusotomia que evoluiu bem. Caso 2) Paciente do sexo feminino, 70 anos, procurou atendimento por história de sinusites de repetição. Ao exame físico não se percebeu nenhuma particularidade. A tomografia computadorizada, assim como a ressonância magnética, detectou espessamento da parede mucosa do seio maxilar esquerdo, além de uma massa calcificada. Realizouse a mesma sequência de tratamento e a paciente também evoluiu bem.

Considerações finais: A infecção fúngica deve ser considerada nos pacientes que se apresentam com sinusite crônica, que não respondem ao uso de antibióticos e que possuem história de manipulação endodôntica.

Palavras-chave: micetoma, sinusite, sinusite maxilar, seios paranasais, aspergillus.

\section{SUMMARY}

Introduction: Fungal ball of the sinuses is a not invasive infection that if characterizes for its chronicity, being the majority related with previous endodontic treatment. Affect mainly the breasts to maxillary; even so all the breasts can be involved. The main etiological agent is the Aspergillus spp. The computed tomography, had to characteristic the radiological presentations, suggests the diagnosis that is carried through definitively through histopathological analyses. The treatment standard-gold is the sinus surgery with average meatal antrostomy.

Objective: Reporting two cases of fungal ball of the sinuses and to stand out important aspects of this pathology.

Story of the Cases: Case 1) Patient of the feminine sex, 78 years old, presented itself with complaints of face pain has 6 months and previous history of endodontic treatment. To the physical examination it was evidenced purulent secretion presence in left average meatus. Ray X presented complete veiling of the breasts to maxillary left, while the computed tomography showed injury calcified in this place. Sinusotomy was become fulfilled that evolved well. Case 2) Patient of the feminine sex, 70 years old, looked attendance for history of sinusitis of repetition. To the physical examination no particularity was not perceived. The computed tomography, as well as the magnetic resonance, detected thickening of the mucous wall of the breasts to maxillary left, beyond a calcified mass. It was become fulfilled same sequence of treatment and the patient also evolved well.

Final Considerations: The fungal infection must be considered in the patients who if present with chronic sinusitis, that they do not answer to the antibiotic use and that they possess history of endodontic manipulation.

Keyword: mycetoma, sinusitis, maxillary sinusitis, sinuses, aspergillus. droaereo and presence of hypo intense of left maxillary sinus. 


\section{INTRODUÇÃO}

Rinossinusite (RS) é uma patologia que reflete um processo inflamatório ou infeccioso da região nasal e contiguamente aos seios paranasais. É uma desordem que afeta anualmente mais de 31 milhões de americanos de todas as idades e gêneros (1). A RS pode ser dividida em aguda (quando dura até 12 semanas) ou crônica (durando mais de 12 semanas), uma vez que não há substrato histopatológico que diferencia a forma aguda da subaguda. Também pode ser classificada em RS viral, bacteriana, fúngica ou alérgica (2).

A frequência de rinossinusite fúngica (RSF), apesar de ser incomum, tem aumentado nas últimas duas décadas (3). Em pacientes com RS crônica, 6 a 12\% irão apresentar fungos na cultura ou em estudo histopatológico (4). Há pouco tempo também se observou que sua classificação é de suma importância para se predizer de forma mais acurada o prognóstico e otimizar de forma efetiva sua terapia. Há dois tipos básicos de RSF: a forma invasiva e a não-invasiva. Além disso, há ainda 5 subtipos distintos dependendo do sistema imunológico do paciente (5): em imunossuprimidos a forma invasiva aguda/fulminante e invasivo crônico/indolente (que pode se manifestar em imunocompetentes também, na forma granulomatosa ou não-granulomatosa); em imunocompetentes, a colonização saprofítica e a bola fúngica; e em atópicos, a sinusite fúngica alérgica.

A presente série de casos abordará dois casos de RSF não invasiva em uma clínica privada de Caxias do Sul, com suas evoluções diagnósticas e seus tratamentos, além de uma breve revisão da literatura sobre a patologia.

\section{Relato dos Casos}

Caso 1) Paciente do sexo feminino, aposentada, com 78 anos, apresentou-se em um consultório particular com queixa de dor malar com irradiação para arcada dentária superior à esquerda há cerca de seis meses. Realizou avaliação com dentista e, apesar de ter tratado endodônticamente o $\mathrm{n}^{\circ} 26$ no passado, foi liberada. Relatou que após exposição a alterações climáticas teve um quadro compatível com infecção de vias aéreas superiores e recebeu um tratamento com antibiótico que aliviou temporariamente os sintomas. Seu único fator de alívio era o uso frequente de antiinflamatório não esteroidal (AINE). A radiografia trazida pela paciente apresentava velamento completo de seio maxilar esquerdo. Ao exame físico e endoscopia nasosinusal, constatou-se um desvio de septo e a presença de secreção purulenta em meato médio esquerdo. Solicitou-se uma Tomografia Computadorizada
(TC) de face que apresentou lesão com calcificação do seio maxilar esquerdo. Foi indicada uma sinusotomia maxilar endoscópica à esquerda com anatomopatológico e cultura específica para fungos (Figura 1). A evolução pós-operatória transcorreu sem intercorrências.

Caso 2) Paciente do sexo feminino, aposentada, de 70 anos, que se apresentou em um consultório particular encaminhada devido a um histórico de sinusites de repetição e rinite alérgica. Estava em uso de furoato de fluticasona spray nasal e não apresentava queixas. Trazia consigo uma TC de face que apresentava espessamento do revestimento mucoso do seio maxilar esquerdo com nível hidroaéreo associado, identificando-se massa com densidade de partes moles junto à parede medial e ao seu antro, com pequenas calcificações de permeio (Figura 2). Demais seios paranasais exibindo aeração e desenvolvimento usuais. Ao exame físico e endoscopia nasosinusal nenhuma particularidade foi encontrada. Solicitou-se uma Ressonância Magnética (RM) de face que se apresentou com hipossinal, acometendo a cavidade maxilar esquerda, a qual exibia ligeiro espessamento parietal, com presença de nível hidroaéreo e, também, estrutura com padrão denso, exibindo componente calcificatório associado (Figura 3). Esse hipossinal à RM com hipertenuação de TC são sinais típicos de bola fúngica. Foi realizado a mesma sequência de tratamento cirúrgico e exames de diagnóstico etiológico. Esta paciente também evoluiu bem.

Ambos os casos apresentaram Aspergillusspp como agente etiológico.

\section{DISCUSSÃO}

Bola fúngica é descrita como um acúmulo não invasivo de uma densa conglomeração de hifas fúngicas em um seio paranasal. É caracterizada por uma massa de restos fúngicos endurecidos e muco, com crescimento progressivo na cavidade sinusal, sem envolvimento da mucosa subjacente (3). Bolas fúngicas foram erroneamente classificadas como micetomas ou aspergilomas no passado.

Durante as últimas décadas tem havido um aparente aumento no diagnóstico dessa patologia (3). Bolas fúngicas foram encontradas em $3,7 \%$ dos pacientes operados na Mayo Clinic por condições inflamatórias crônicas dos seios paranasais (6). A incidência pode estar artificialmente aumentada de inúmeras formas, incluindo a melhordetecção através de avaliações radiológicas e maior expectativa de vida (7).

A idade média de acometimento é 64 anos, tendo o sexo feminino uma predominância de $64 \%$, condizendo 


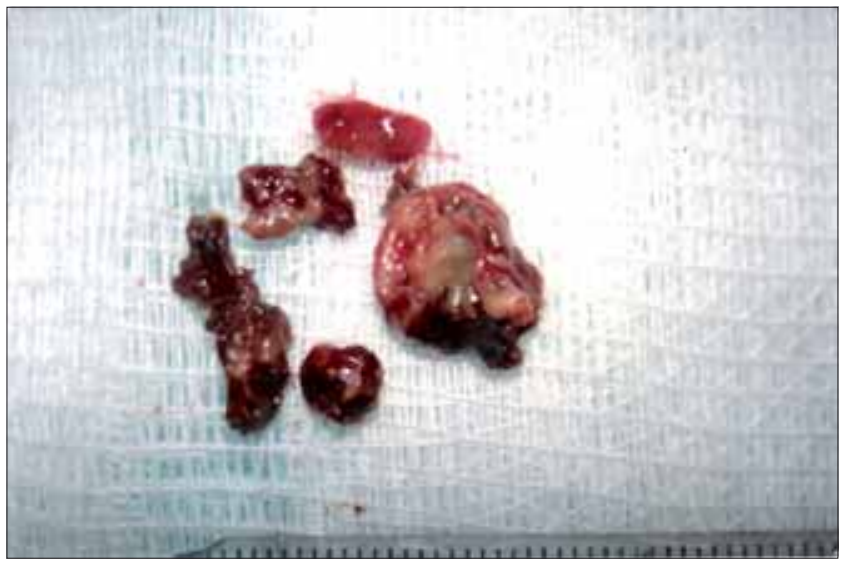

Figura 1. Peça cirúrgica pós sinusotomia maxilar.

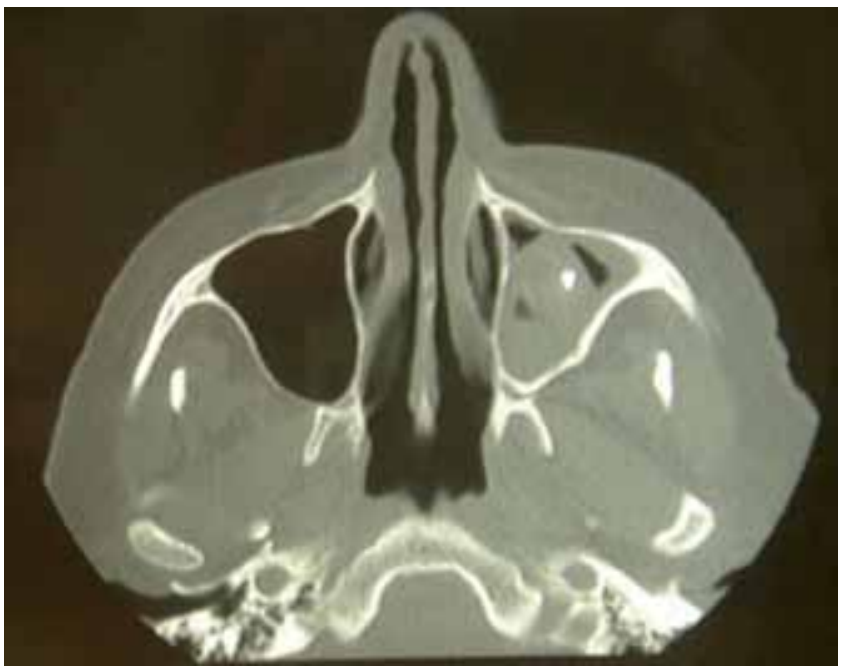

Figura 2. TC de face apresentando espessamento do revestimento mucoso do seio maxilar esquerdo e massa de densidade de partes moles com calcificações.

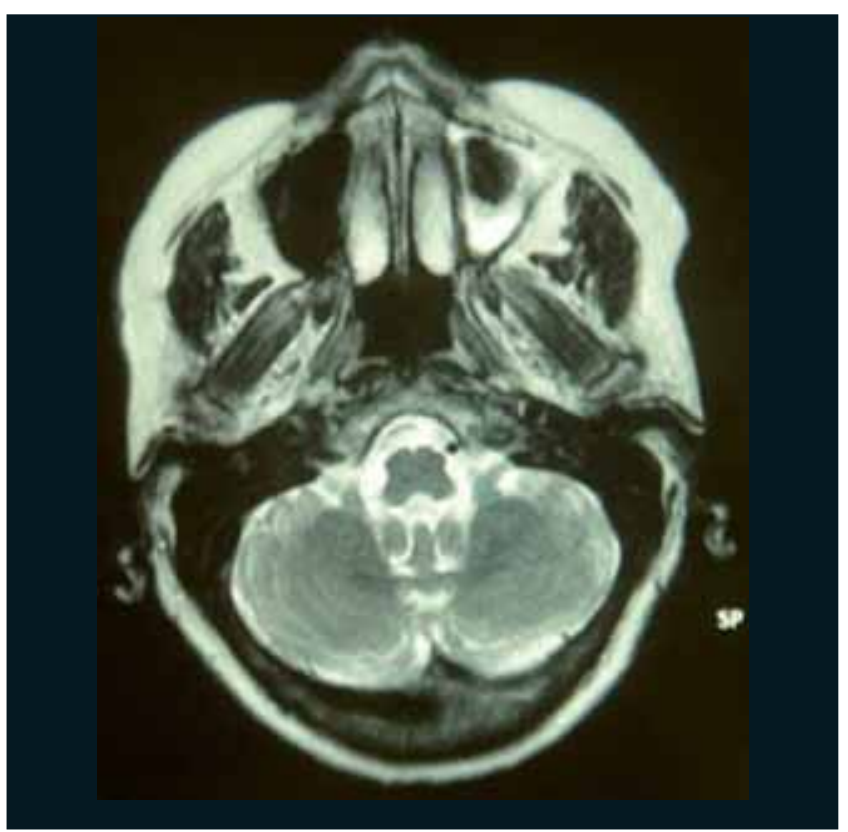

Figura 3. RM de face com nível hidroaéreo e presença de hipossinal de seio maxilar esquerdo. com as características dos casos relatados. Os indivíduos são tipicamente imunocompetentes. O local mais frequentemente envolvido é o seio maxilar, seguido pelo seio esfenoide (8). O caso 1 apresentou um histórico de tratamento dentário prévio, estando esta característica presente em cerca de $84 \%$ dos pacientes diagnosticados com bola fúngica (6). Em um estudo de caso-controle, o risco de desenvolver a doença em pacientes submetidos a tratamento endodôntico foi 14 vezes maior que o dos pacientes sem tratamento (9).

A fisiopatologia da bola fúngica ainda não é totalmente compreendida, existindo várias teorias para tentar explicar seu aparecimento. A teoria aerogênica postula que os esporos inalados de fungo se depositariam nos seios paranasais, tornando-se patogênicos quando as condições no interior dos seios se tornassem relativamente anaeróbicas. Outra teoria sugere que a obstrução funcional do óstio sinusal poderia agir como um fator indutor, e que o crescimento fúngico poderia ser favorecido pelas condições hipóxicas e anaeróbicas. Além disso, a diminuição da ventilação diminuiria o $\mathrm{pH}$, favorecendo o crescimento das hifas (10). Um outro mecanismo proposto conjecturou que o crescimento das hifas pode se estender por um longo período de tempo, com episódios de proliferação alternando com períodos mais longos de inatividade. Superinfecções poderiam levar a episódios de sinusite aguda, e a secreção purulenta resultante consistiria em um meio nutritivo ideal para potencializar o crescimento fúngico. Por fim, existe a hipótese da passagem de selantes dentários contendo óxido de zinco aos seios maxilares poderia ser um fator indutor importante de alterações necróticas e inflamatórias na mucosa, o que favoreceria o crescimento fúngico (11). Esta última hipótese, no entanto, não explica o aparecimento de bolas fúngicas em outros seios paranasais.

A bola fúngica pode ser assintomática, como ocorreu no primeiro caso, ou pode simular quadros de rinossinusite crônica (7). Sintomas frequentes incluem obstrução nasal, secreção nasal purulenta, cacosmia e dor facial. Sintomas menos comuns incluem convulsões, epistaxe, proptose, febre, tosse e visão borrada. Os sintomas geralmente duram um longo período de tempo e podem estar presentes por meses ou até anos (8).

O diagnóstico de bola fúngica deve ser considerado nos casos de sinusites recorrentes ou refratárias aos tratamentos com antibióticos previamente utilizados (caso 2) e especialmente quando forem unilaterais $(6,8)$. Evidências radiológicas, através de radiografias e, principalmente, TC sugerem fortemente este diagnóstico (11). Entretanto, o diagnóstico definitivo é baseado nas características macroscópicas e histopatológicas da peça cirúrgica $(6,8)$. Frequentemente, as culturas a presentam resultados negativos, sendo que em apenas $23-50 \%$ dos casos as culturas 
positivam (8). Destes, a espécie Aspergillus fumigatus cresce em mais de $90 \%$ dos casos (12). Nos casos relatados, o agente etiológico responsável foi o Aspergillusspp. Após propor alguns critérios para diagnóstico e compará-los com o achado em 20 casos publicados na literatura médica, DESHAzo et al chegou a cinco critérios para o diagnóstico de micetoma dos seios paranasais (13) (Tabela1).

O exame de imagem de escolha para o diagnóstico de micetoma é a TC. O achado mais comum neste exame é a opacificação completa ou parcial do seio acometido. Microcalcificações ou manchas com densidade metálica também são encontrados em cerca de um terço dos casos de opacificação de um seio (6).

A melhor forma de tratamento de micetoma de seios paranasaisé a cirurgia, visto que as drogas antifúngicas, tópicas ou sistêmicas, não estão indicadas (14), exceto nos casos de imunodepressão, em que alguns autores recomendam tratamento adjuvante com o uso de antifúngicos sistêmicos, como itraconazol (8).

Duas formas de procedimentos cirúrgicos para remoção da bola fúngica no seio maxilar estão descritas na literatura médica: o histórico procedimento de CaldwellLuc, que consiste na abertura da fossa canina para obtenção de acesso ao seio maxilar; além da cirurgia por via endoscópica, padrão-ouro atualmente (14). Os dois casos relatados neste trabalho foram tratados com sinusotomia porvia endoscópica.

A abordagem dos seios paranasais via endoscópica visa à abertura natural do óstio do seio acometido, no intuito de recuperar a sua drenagem e ventilação. Caso haja mucosa polipoide junto ao meato médio um debridamento pode ser realizado. Além disso, uma larga antrostomia pode ser criada removendo grande parte da fontanela posterior e conectando um possível óstio secundário à área do óstio maxilar natural. Lavagens com aspirador devem ser realizadas ao longo do procedimento (14).

Este procedimento causa menos danos ao paciente, bem como restabelece a ventilação e a drenagem do seio ao seu óstio natural (14). Por outro lado, esta cirurgia apresenta um problema técnico: não é possível visualizar o ângulo formado pelo osso do ducto lacrimal e a parede anterior do seio maxilar. Neste ponto, pode permanecer um concentrado de partículas fúngicas após a cirurgia, o que pode gerar recidivas (15).

Quando a bola fúngica acomete o seio esfenoidal ou o etmoidal, a abordagem de escolha é, respectivamente, esfenoidotomia endonasal ou etmoidectomia completa. Já nos raros casos onde o seio comprometido é o frontal um acesso endonasal geralmente é possível (14).
Tabela I. Critérios clinico-patológicos propostos para o diagnóstico de micetoma dos seios paranasais

I) Evidência de opacificação do seio comou sem calcificações floculentas.

2) Conteúdo mucopurulento, fétido ou com coloração semelhante à argila dentro de um seio paranasal.

3) Uma conglomeração densa de hifas emaranhadas, mas adjacente à mucosa respiratória do seio .

4) Resposta inflamatória crônica de intensidade variável na mucosa adjacente aos elementos fúngicos. Esta resposta inclui linfócitos, células do plasma, mastócitos e eosinófilos, sem predominância de eosinófilos ou respostagranulomatosa. Mucina alérgica está ausente na coloração de hematoxilinaeosina.

5) Semevidência histológica de invasão fúngica da mucosa, associada a vasos sanguíneos ou ao osso adjacente, visualizado microscopicamente com colorações especiais para fungos.

Adaptado de deSHAZO etal, 1997( I3).

\section{COMENTÁRIOS FINAIS}

Apesar do avanço dos métodos diagnósticos e dos exames de imagem, o diagnóstico de sinusite fúngica ainda permanece um desafio. A infecção fúngica deve ser considerada em todos os pacientes com sinusite crônica, quando não há resposta terapêutica adequada ou em sinusites unilaterais recorrentes, sendo mais presentes naqueles submetidos a tratamentos endodônticos prévios. Considerado um diagnóstico de exclusão, muitas vezes os pacientes acometidos chegam ao consultório já passaram por mais de um profissional de saúde, como os casos relatados. Nesses casos, uma TC de seios paranasais deve ser considerada, mas seu diagnóstico definitivo é baseado em análises macroscópicas e histopatológicas da peça cirúrgica.

\section{REFERÊNCIAS BIBLIOGRÁFICAS}

1. Infectious rhinosinusitis in adults: classification, etiology and management. International Rhinosinusitis Advisory Board. Ear Nose Throat J. 1997, 76(12 Suppl):1-22.

2. Associação Brasileira de Otorrinolaringologia e Cirurgia Cérvico-Facial. Diretrizes Brasileiras de Rinossinusites (suplemento). Br J Otorhinolaryngol. 2008, 74(2).

3. Nicolai P, Lombardi D, Tomenzoli D, Villaret AB, Piccioni $\mathrm{M}$, Mensi M, et al. Fungus ball of the paranasal sinuses: experience in 160 patients treated with endoscopic surgery. Laryngoscope. 2009, 119(11):2275-9. 
4. Taxy JB. Paranasal fungal sinusitis: contributions of histopathology to diagnosis: a report of 60 cases and literature review. Am J Surg Pathol. 2006, 30(6):713-20.

5. Ferguson BJ. Definitions of fungal rhinosinusitis. Otolaryngol Clin North Am. 2000, 33(2):227-35.

6. Grosjean P, Weber R. Fungus balls of the paranasal sinuses: a review. Eur Arch Otorhinolaryngol. 2007, 264(5):461-70.

7. Robey AB, OBrien EK, Richardson BE, Baker JJ, Poage DP, LeopoldDA. The changing face of paranasal sinus fungus balls. Ann Otol Rhinol Laryngol. 2009, 118(7):500-5.

8. Ferguson BJ. Fungus balls of the paranasal sinuses. Otolaryngol Clin North Am. 2000, 33(2):389-98.

9. Mensi M, Piccioni M, Marsili F, Nicolai P, Sapelli PL, Latronico $\mathrm{N}$. Risk of maxillary fungus ball in patients with endodontic treatment on maxillary teeth: a case-control study. Oral Surg Oral Med Oral Pathol Oral Radiol Endod. 2007, 103(3):433-6.

10. Stevens MH. Primary fungal infections of the paranasal sinuses. Am J Otolaryngol. 1981, 2(4):348-57.
11. Stammberger H, Jakse R, Beaufort F. Aspergillosis of the paranasal sinuses $\mathrm{x}$-ray diagnosis, histopathology, and clinical aspects. Ann Otol Rhinol Laryngol. 1984, $93(3 \mathrm{Pt}$ 1):251-6.

12. Klossek JM, Serrano E, Peloquin L, Percodani J, Fontanel JP, Pessey JJ. Functional endoscopic sinus surgery and 109 mycetomas of paranasal sinuses. Laryngoscope. 1997, 107(1):112-7.

13. deShazo RD, OBrien M, Chapin K, Soto-Aguilar M, Swain $\mathrm{R}$, Lyons $\mathrm{M}$, etal. Criteria for the diagnosis of sinus mycetoma. J Allergy Clin Immunol. 1997, 99(4):475-85.

14. Costa F, Polini F, Zerman N, Robiony M, Toro C, Politi M. Surgical treatment of Aspergillus mycetomas of the maxillary sinus: review of the literature. Oral Surg Oral Med Oral Pathol Oral Radiol Endod. 2007, 103(6):e23-9.

15. Chobillon MA, Jankowski R. What are the advantages of the endoscopic canine fossa approach in treating maxillary sinus aspergillomas? Rhinology. 2004, 42(4):230-5. 